\title{
Roles of arabidopsis WRKY18, WRKY40 and WRKY60 transcription factors in plant responses to abscisic acid and abiotic stress
}

\author{
Han Chen ${ }^{1}$, Zhibing Lai', Junwei Shi ${ }^{1}$, Yong Xiao ${ }^{1}$, Zhixiang Chen ${ }^{2}$, Xinping Xu ${ }^{1 *}$
}

\begin{abstract}
Background: WRKY transcription factors are involved in plant responses to both biotic and abiotic stresses. Arabidopsis WRKY18, WRKY40, and WRKY60 transcription factors interact both physically and functionally in plant defense responses. However, their role in plant abiotic stress response has not been directly analyzed.

Results: We report that the three WRKYs are involved in plant responses to abscisic acid (ABA) and abiotic stress. Through analysis of single, double, and triple mutants and overexpression lines for the WRKY genes, we have shown that WRKY18 and WRKY60 have a positive effect on plant ABA sensitivity for inhibition of seed germination and root growth. The same two WRKY genes also enhance plant sensitivity to salt and osmotic stress. WRKY40, on the other hand, antagonizes WRKY18 and WRKY60 in the effect on plant sensitivity to ABA and abiotic stress in germination and growth assays. Both WRKY18 and WRKY40 are rapidly induced by ABA, while induction of WRKY60 by ABA is delayed. ABA-inducible expression of WRKY60 is almost completely abolished in the wrky 18 and wrky 40 mutants. WRKY18 and WRKY40 recognize a cluster of W-box sequences in the WRKY60 promoter and activate WRKY60 expression in protoplasts. Thus, WRKY60 might be a direct target gene of WRKY18 and WRKY40 in ABA signaling. Using a stable transgenic reporter/effector system, we have shown that both WRKY18 and WRKY60 act as weak transcriptional activators while WRKY40 is a transcriptional repressor in plant cells.
\end{abstract}

Conclusions: We propose that the three related WRKY transcription factors form a highly interacting regulatory network that modulates gene expression in both plant defense and stress responses by acting as either

transcription activator or repressor.

\section{Background}

Plants are constantly exposed to a variety of biotic and abiotic stresses and have evolved intricate mechanisms to sense and respond to the adverse conditions. Phytohormones such as salicylic acid (SA), ethylene (ET), jasmonic acid (JA) and abscisic acid (ABA) play important roles in the regulation of plant responses to the adverse environmental conditions. In Arabidopsis, mutants deficient in SA biosynthesis (e.g. sid2) or signalling (e.g. npr1) exhibit enhanced susceptibility to biotrophic pathogens, which parasitize on plant living tissue $[1,2]$. ET- and JA-mediated signaling pathways, on the other

\footnotetext{
* Correspondence: Issxxp@mail.sysu.edu.cn

${ }^{1}$ State Key Laboratory of Biocontrol and Key Laboratory of Gene Engineering of the Ministry of Education, School of Life Sciences, Sun Yat-sen University, Guangzhou 510275, China

Full list of author information is available at the end of the article
}

hand, often mediate plant defense against necrotrophic pathogens that promote host cell death at early stages of infection [3]. ABA is extensively involved in plant responses to abiotic stresses including drought, extreme temperatures and osmotic stress $[4,5]$. ABA also plays a regulatory role in important plant growth and developmental processes including seed development, dormancy, germination and stomatal movement. Recent studies have reported crosstalk of signaling pathways regulated by these signal molecules that contributes to either antagonistic or synergistic interactions between abiotic and biotic interactions [6,7].

A large body of evidence indicates that plant WRKY DNA-binding transcription factors play important role in plant defense responses. In Arabidopsis, a majority of its WRKY genes are induced by pathogen infection or SA treatment [8]. A large number of plant defense or 
defense related genes including pathogenesis-related $(P R)$ genes and the regulatory NPR1 gene contain W box sequences in their promoters that are recognized by WRKY proteins [9]. A number of studies have shown that these W-box sequences are necessary for the inducible expression of these defense genes. Mutant analyses in Arabidopsis have revealed direct links between specific WRKY proteins and complex plant defense responses. Mutations of WRKY70 enhance plant susceptibility to both biotrophic and necrotrophic pathogens including Erwinia carotovora, Hyaloperonospora parasitica, Erysiphe cichoracearum and Botrytis cinerea [10-12]. Disruption of WRKY33 results in enhanced susceptibility to necrotrophic fungal pathogens and impaired expression of JA/ET-regulated defense genes [13]. Mutations of other WRKY genes including WRKY7, WRKY11, WRKY17, WRKY48, WRKY38 and WRKY62, on the other hand, enhance basal plant resistance to virulent $P$. syringae strains, suggesting that they function as negative regulators of plant basal defense [14-17].

There is also evidence that WRKY transcription factors are involved in plant responses to abiotic stresses. Microarray experiments have identified WRKY genes that are induced by various abiotic stresses. In Arabidopsis, for example, WRKY genes were among several families of transcription factor genes that are induced by drought, cold or high-salinity stress [18-20]. The barley $H v-W R K Y 38$ gene is rapidly and transiently induced during exposure to low non-freezing temperature in ABA-independent manner and exhibits continuous induction during dehydration and freezing treatment [21]. In tobacco, a WRKY transcription factor is specifically induced during a combination of drought and heat shock [22]. Regulated expression of WRKY genes during plant stress responses provides circumstantial evidence that implicates WRKY proteins in plant responses to abiotic stress. In Creosote bush (Larrea tridentate) that thrives in vast arid areas of North American, a WRKY protein (LtWRKY21) is able to activate the promoter of an ABA-inducible gene, HVA22, in a dosage-dependent manner [23]. A number of rice WRKY proteins regulate positively or negatively ABA signalling in aleurone cells $[23,24]$. Overexpression of soybean GmWRKY13, GmWRKY21 and GmWRKY54 conferred differential tolerance to abiotic stresses in transgenic Arabidopsis plants [25]. However, stable or transient overexpression of a gene in transgenic plants can often lead to pleiotropic phenotypes that may or may not reflect the true biological functions of the gene. Very recently, Jiang and $\mathrm{Yu}$ [26] have reported that Arabidopsis wrky2 knockout mutants are hypersensitive to ABA responses during seed germination and postgermination early growth, suggesting an important role of the stress-regulated WRKY gene in plant stress responses.
Arabidopsis WRKY18, WRKY4O and WRKY60 are pathogen-induced and encode three structurally related WRKY proteins [27]. We have previously shown that WRKY18, WRKY40 and WRKY60 interact physically with themselves and with each other through a leucinezipper motif at their N-terminus [27]. Analysis with both knockout alleles and overexpresison lines indicated that the three pathogen-induced WRKY transcription factors have a partially redundant negative effect on SAmediated defense but exerted a positive role in JAmediated defense. [27]. Likewise, ABA plays a complex role in plant defense response. In Arabidopsis, ABA counteracts SA-dependent defense against the hemibitrophic bacterial pathogen Pseudomonas syringae [7], but is a signal required for resistance to the necrotrophic pathogens Pythium irregulare and Alternaria brassicicola [28]. In the present study, we report that Arabidopsis WRKY18, WRKY40 and WRKY60 proteins indeed function in a complex pattern in plant responses to ABA and abiotic stresses. The complex roles of the three WRKY transcription factors in plant biotic and abiotic stress responses are consistent with the complex nature of their expression, transcription-regulating activities and physical interactions.

\section{Results}

\section{Altered ABA Sensitivity of Mutants and Overexpression Plants}

To determine their possible roles in plant $A B A$ response, we first performed germination experiments to analyze the ABA sensitivity of previously characterized knockout mutants and overexpression lines for WRKY18, WRKY40 and WRKY60 (Figure 1; Additional file 1). In the absence of $A B A, 100 \%$ of wild-type seeds and more than $85 \%$ of WRKY18-overexpressing plants germinated (Figure 1A). In the presence of 0.5 and 1.0 $\mu \mathrm{M}$ ABA, however, the germination rates of WRKY18overexpressing plants were reduced to $50 \%$ and $20 \%$ of those of wild type, respectively (Figure 1A). At $1.5 \mu \mathrm{M}$ ABA, germination of WRKY18-overexpression plants was completely inhibited while almost $80 \%$ of wild-type seeds still germinated (Figure 1A). Thus, overexpression of WRKY18 enhanced seed sensitivity to ABA in germination assays. Disruption of WRKY18, on the other hand, significantly reduced plant sensitivity to $A B A$ as indicated by an approximate $15 \%$ increase in the germination rates of the wrky18 mutant at 1.0, 1.5 and 2.0 $\mu \mathrm{M}$ ABA over those of wild-type plants (Figure 1A). Thus disruption of WRKY18 reduced seed sensitivity to $A B A$ in germination assays. Similar results were observed for WRKY60 from the germination experiments. In the absence of $A B A$, the germination rates of both the knockout mutant and overexpression line for WRKY60 were similar to those of wild type (Figure 1C). 

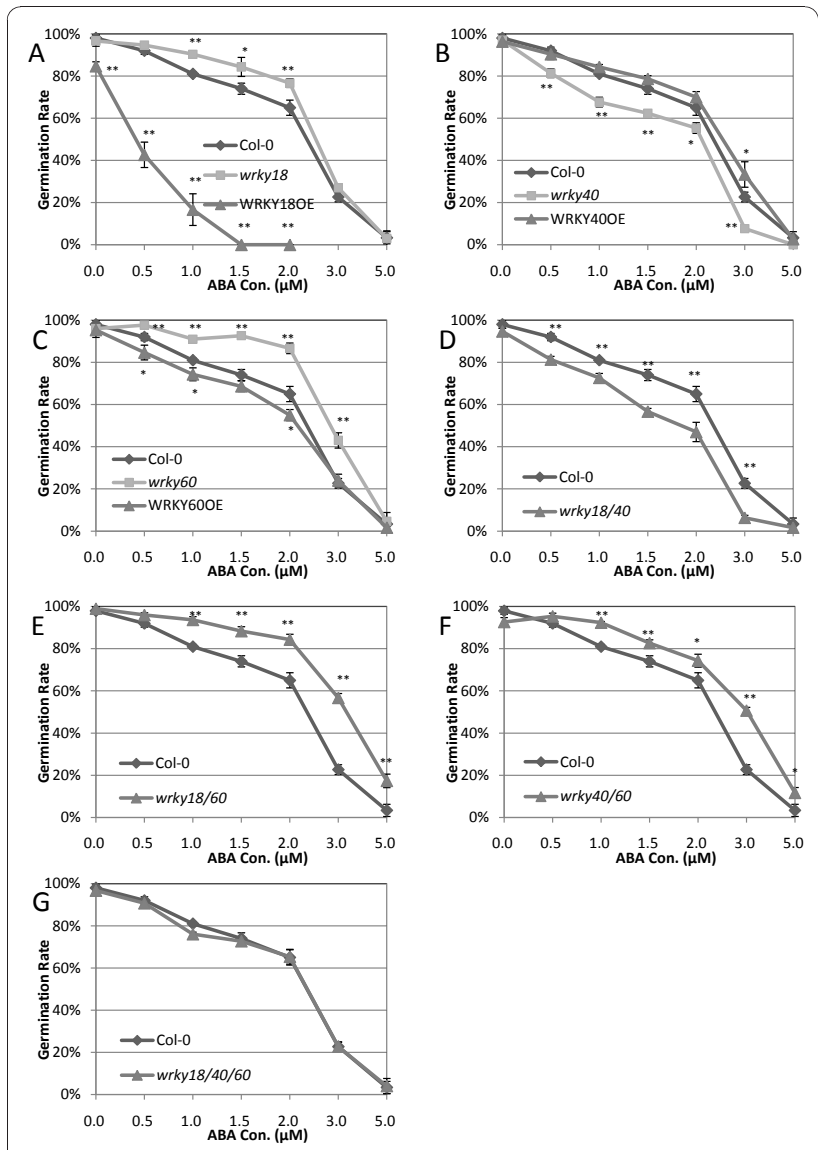

Figure 1 Altered germination rates under exogenous ABA treatment. Seeds of wild type, mutants and overexpression lines were sown on 1/2 MS media containing indicated concentrations of ABA. Seedlings with green cotyledons were considered as germinated. Germination rates were determined 120 hours after sowing. The means and standard errors were calculated from three independent experiments. (Asterisks: p-value $<0.05$; Double

Asterisks: $p$-value $<0.01$ )

When ABA was added to the medium, germination of the wrky60 mutant was less inhibited than that of wild type. For example, when ABA concentration was increased from 0 to $2 \mu \mathrm{M}$, there was only about $10 \%$ reduction in germination rate of the wrky60 mutant compared to more than $40 \%$ reduction of wild type (Figure 1C). Furthermore, overexpression of WRKY60 enhanced plant ABA sensitivity as indicated by significantly increase in inhibition of germination in the overexpression line relative to that of wild type (Figure 1C). Increased inhibition of germination in the WRKY60overexpressing lines, however, was much less than that in the WRKY18-overexpressing line (Figure 1A, C). By contrast, the wrky40 knockout mutant was more sensitive and the overexpression line was less sensitive than wild type to the inhibitory effect of $\mathrm{ABA}$ on germination (Figure 1B).
We have previously shown that structurally related WRKY18, WRKY40 and WRKY60 interact both physically and functionally in the regulation of plant basal defense [27]. To determine possible functional interactions among the three WRKY proteins, we compared the ABA sensitivity of their double and triple knockout mutants (Figure 1D, E, G and 1F; Additional file 1). Germination rates of the wrky18 wrky60 double mutant at relatively low ABA concentrations $(<2 \mu \mathrm{M})$ were higher than those of wild type and were similar to those of the wrky60 single mutant (Figure 1E). At higher ABA concentrations ( 3 and $5 \mu \mathrm{M}$ ), however, the germination rates of the double mutant were $10-15 \%$ higher than those of the wrky60 single mutant (Figure 1E). Thus, WRKY18 and WRKY60 act additively in enhancing seed sensitivity to ABA in germination assays. The germination rates of the wrky18 wrky40 double mutant at various ABA concentrations were substantially lower than those of wild type and the wrky 40 single mutant (Figure 1D). Interestingly, the germination rates of the wrky40 wrky60 double mutant were significantly higher than those of wild type. However, at certain ABA concentrations (e.g. 1.5 and $2.0 \mu \mathrm{M}$ ) the wrky 40 wrky60 double mutant didn't germinate as well as the wrky60 single mutant (Figure 1). There was no significant difference between wild type and the wrky18 wrky 40 wrky60 triple mutant in germination at the various ABA concentrations tested (Figure 1).

We also compared the loss-of-function mutants for ABA-inhibited root growth. When compared with wild type, these mutants had similar root elongation in the absence of ABA (Figure 2A). In the presence of $2 \mu \mathrm{M}$ $\mathrm{ABA}$, root elongation of the wrky18 and wrky60 single mutants and the wrky18 wrky60 double mutant was less inhibited while the wrky 40 mutant was slightly but not statistically significantly more inhibited than that of wild type (Figure 2). Root elongation of wrky18 wrky40, wrky 40 wrky 60 double mutants and wrky18, wrky 40 wrky60 triple mutant was similar to that of wild type (Figure 2).

\section{Altered tolerance of mutants and overexpression plants to abiotic stress}

$\mathrm{ABA}$ is involved in plant responses to ionic and osmotic stresses. Since the wrky18, wrky 40 and wrky60 mutants exhibited altered sensitivity to ABA in germination assays, we examined root growth of these mutants in growth media containing -0.75 MPa PEG, $200 \mathrm{mM}$ mannitol or $150 \mathrm{mM} \mathrm{NaCl}$. In the normal growth media, root elongations of all the mutants were similar to that of wild type (Figure 2). After transfer to the growth media containing PEG, mannitol or $\mathrm{NaCl}$, the wrky18, wrky60 single mutants and wrky18 wrky60 double mutant was less sensitive than wild type to the 

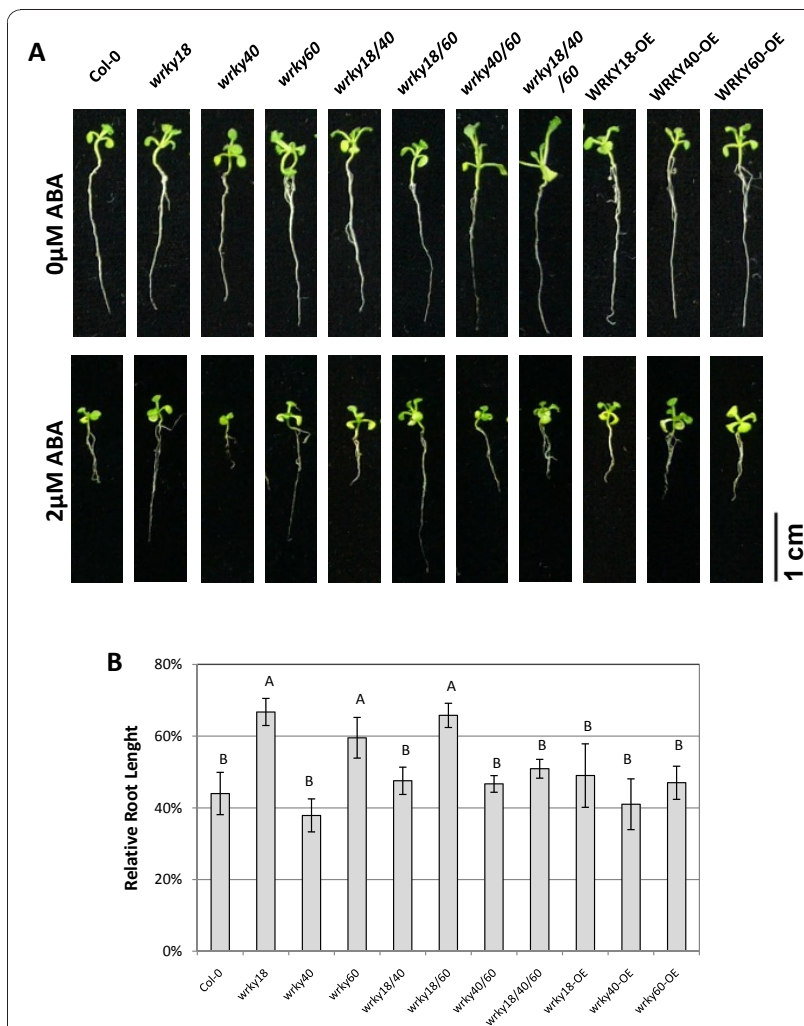

Figure 2 Altered root elongation under exogenous $A B A$ treatment. Seeds of wild type and mutants were grown on 1/2 MS media for four days and then were transferred to MS agar media containing 0 or $2 \mu \mathrm{M}$ ABA. The picture was taken and the root length was determined at the 7th day after the transfer. The relative root length was the ratio of average root length of seedlings in $2 \mu \mathrm{M}$ ABA medium to those in $0 \mu \mathrm{M}$ ABA medium. Standard errors were calculated from three independent experiments, every of which employed more than 25 seedlings of each genotype. Groupings were based on Student-Newman-Keuls Test, $\mathrm{a}=0.05$.

osmotic and salt stress conditions (Figure 3; Additional file 2). Root elongation of the wrky18 wrky40 and wrky40 wrky60 double mutants and wrky18 wrky40 wrky60 triple mutant was similar to that of wild type under the osmotic and salt stress conditions (Figure 3; Additional file 2).

\section{Induced expression by $A B A$ and abiotic stress}

WRKY18, WRKY40 and WRKY60 are induced in Arabidopsis plants upon infection by pathogen infection and SA [27]. Because of their role in plant response to ABA and abiotic stresses, we performed quantitative RT-PCR to analyze the effects of $\mathrm{ABA}$ and abiotic stresses on expression of these three WRKY genes. For determining ABA-regulated expression, we spraying three-week-old plants with $5 \mu \mathrm{M}$ ABA and examined the transcript levels of the WRKY genes at 0 to 24 hours after the treatment. As shown in Figure 4A, the levels of WRKY18 and WRKY4O transcripts increased by about

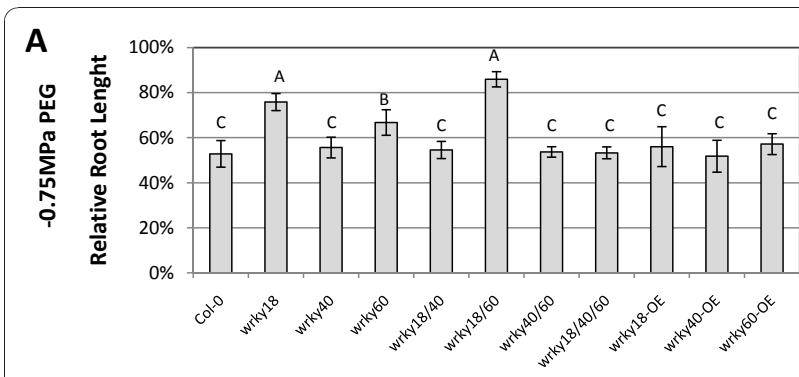

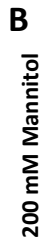
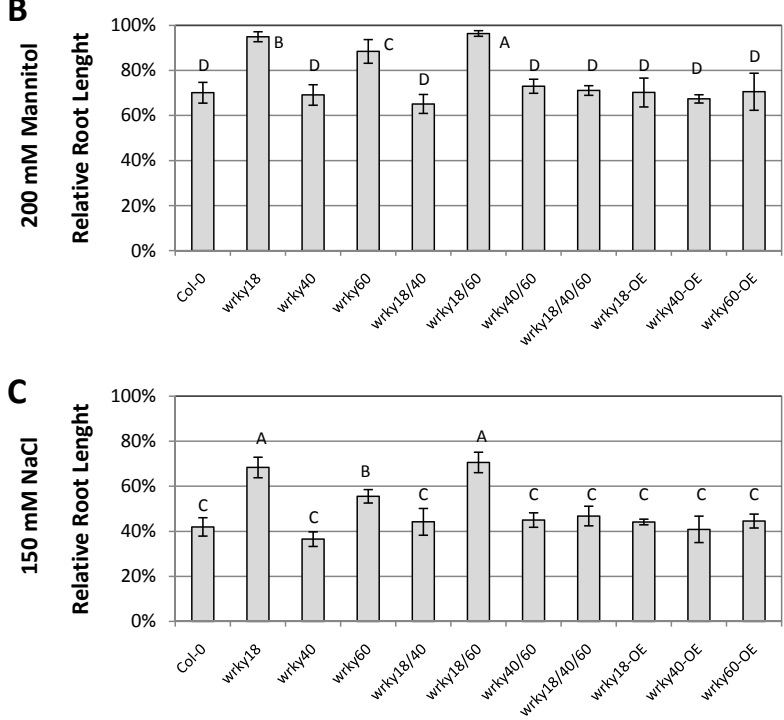

Figure 3 Altered stress tolerance of the WRKY mutants. Seeds of wild type and mutants were grown on 1/2 MS media for four days and then were transferred to MS agar media without or with -0.75 MPa PEG, $200 \mathrm{mM}$ mannitol or $150 \mathrm{mM} \mathrm{NaCl}$. The picture was taken and the root length was determined at the 7th day after the transfer. The average root length of each genotype in MS medium and their standard errors were calculated from three independent experiments, every of each employed more than 25 seedlings per genotype. Relative root length was the ratio of average root lengths of seedlings in medium with $200 \mathrm{mM}$ mannitol, $-0.75 \mathrm{MPa}$ PEG or $150 \mathrm{mM} \mathrm{NaCl}$ to those in MS medium. The standard errors were calculated from three independent experiments, every of each employed more than 25 seedlings per genotype. Groupings were based on Student-Newman-Keuls Test, $\mathrm{a}=005$.

10 and 16 fold during the first hour after ABA treatment, respectively. After 12 hours of ABA treatment, however, the transcript levels for both WRKY18 and WRKY40 were back to basal levels (Figure 4A), indicating that induction of the two WRKY genes by ABA was transient. By contrast, no significant increase in the transcript level of WRKY60 was observed after the first hour of ABA treatment. By 12 hours after the ABA treatment, the transcript level of WRKY60 was increased by about 10 fold above those of control plants (Figure 4A). The elevated levels of WRKY60 transcripts were still substantial even at 24 hour after the ABA treatment (Figure 4A). Thus, induction of WRKY6O by ABA was 


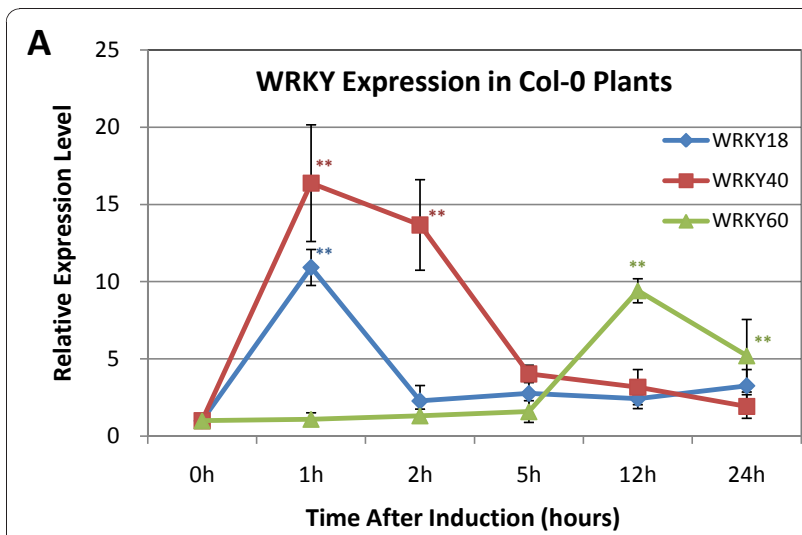

B

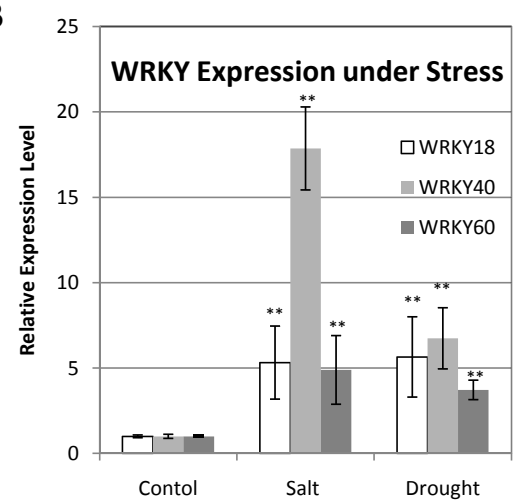

Figure 4 Induced expression of WRKY genes by ABA and abiotic stresses. A. Three-weeks-old wild-type plants were sprayed with water (Mock) or $5 \mu \mathrm{M}$ ABA. Leaves from four treated plants were harvested at indicated time after the treatment for isolation of total RNA and analysis of transcripts using qRT-PCR. Expression level was defined as the ratio of QRT-PCR result of treated sample to its respective mock. The means and standard errors were calculated from three independent experiments. Asterisks mark statistically significant differences of expression level between ABA-treatedleaves harvested immediately and after indicated time. (Asterisks: p-value < 0.05; Double Asterisks: p-value < 0.01; by StudentNewman-Keuls Test). B. One-week-old wild-type seedlings were transferred to1/2 MS media without or with $150 \mathrm{mM} \mathrm{NaCl}$ or -0.75 MPa PEG. The seedlings were collected 24 hours after the transfer for total RNA isolation and analysis of transcripts using qRT-PCR. The means and standard errors were calculated from three independent experiments, all of which included no less than 20 seedlings per sample. Asterisks mark statistically significant differences of expression level between genotypically identical seedlings with or without indicated treatment. (Asterisks: p-value $<0.05$; Double Asterisks: $p$-value < 0.01; by Student-Newman-Keuls Test).

delayed but prolonged when compared to that of WRKY18 and WRKY4O.

We also analyzed responses of the three WRKY genes to salt and drought(PEG) treatments. Wild-type seedlings (7 days old) were transferred to a MS growth medium with or without $150 \mathrm{mM} \mathrm{NaCl}$ or $250 \mathrm{~g} / \mathrm{l}$ PEG and the seedlings were harvested 24 hours later for isolation of total RNA and qRT-PCR analysis. As shown in Figure 4B, the transcript levels for WRKY18, WRKY4O and WRKY60 were elevated by the $\mathrm{NaCl}$ treatment $6.5,18.7$ and 4.9 fold, respectively. After PEG treatment, the three WRKY genes were also induced 4 to 7 fold (Figure 4B). These results indicated that the three WRKY genes were also responsive to abiotic stresses. Induced expression of the WRKY genes by ABA and abiotic stresses have also been observed from previously reported microarray analysis $[29,30]$.

We have previously shown that pathogen-regulated WRKY genes are rich in W boxes in their promoters, suggesting that defense-regulated expression of WRKY genes involve extensive transcriptional activation or repression by its own members of the transcription factor family [8]. To examine possible mutual regulation among the three WRKY genes, we compared wild type and knockout mutants for ABA-regulated expression of the three WRKY genes. As described earlier, WRKY18 was rapidly and transiently induced by ABA in wild-type plants. A similarly rapid and transient induction of WRKY18 was observed in the wrky40 and wrky60 single mutants (Figure 5A). In the wrky 40 wrky60 double mutant, induction of WRKY18 by ABA was also rapid and transient but the magnitude of induction was $2-3$ times higher than those of wild type and their parental single mutants (Figure 5A). Thus, WRKY40 and WRKY60 appear to play cooperatively a negative role in the induction of WRKY18. The levels WRKY4O transcripts also peaked at 1 hour after ABA treatment as observed for WRKY18 but the decline of WRKY transcripts after the first hour was somewhat slower than that of WRKY18 (Figure 5B). In addition, ABA induction of WRKY40 was slightly reduced in the wrky18 and wrky60 mutants (Figure 5B). Thus, WRKY18 and WRKY60 modulate positively induced expression of WRKY4O by ABA.

Induction of WRKY60 by ABA was relatively slow when compared to that of WRKY18 and WRKY4O (Figure 4A). In wild type, no significant induction of WRKY60 transcripts was observed during the first five hours after ABA treatment. However, WRKY60 transcripts increased about 10 fold by 12 hours after the treatment and then declined gradually during the remaining period of the experiments (Figure 4A). In the wrky18 mutant, the induction of WRKY60 was drastically reduced, with only a small increase observed after 24 hours of treatment (Figure $5 \mathrm{C}$ ). In the wrky40 single mutant and wrky 18 wrky 40 double mutant, ABA induction of WRKY60 was completely abolished (Figure 5C). Thus both WRKY18 and WRKY40 are necessary for ABA-induced WRKY60 expression.

\section{Recognition of WRKY 60 promoter by WRKY18 and WRKY40}

Expression analysis using qRT-PCR showed that induction of WRKY18 and WRKY4O by ABA preceded that of 

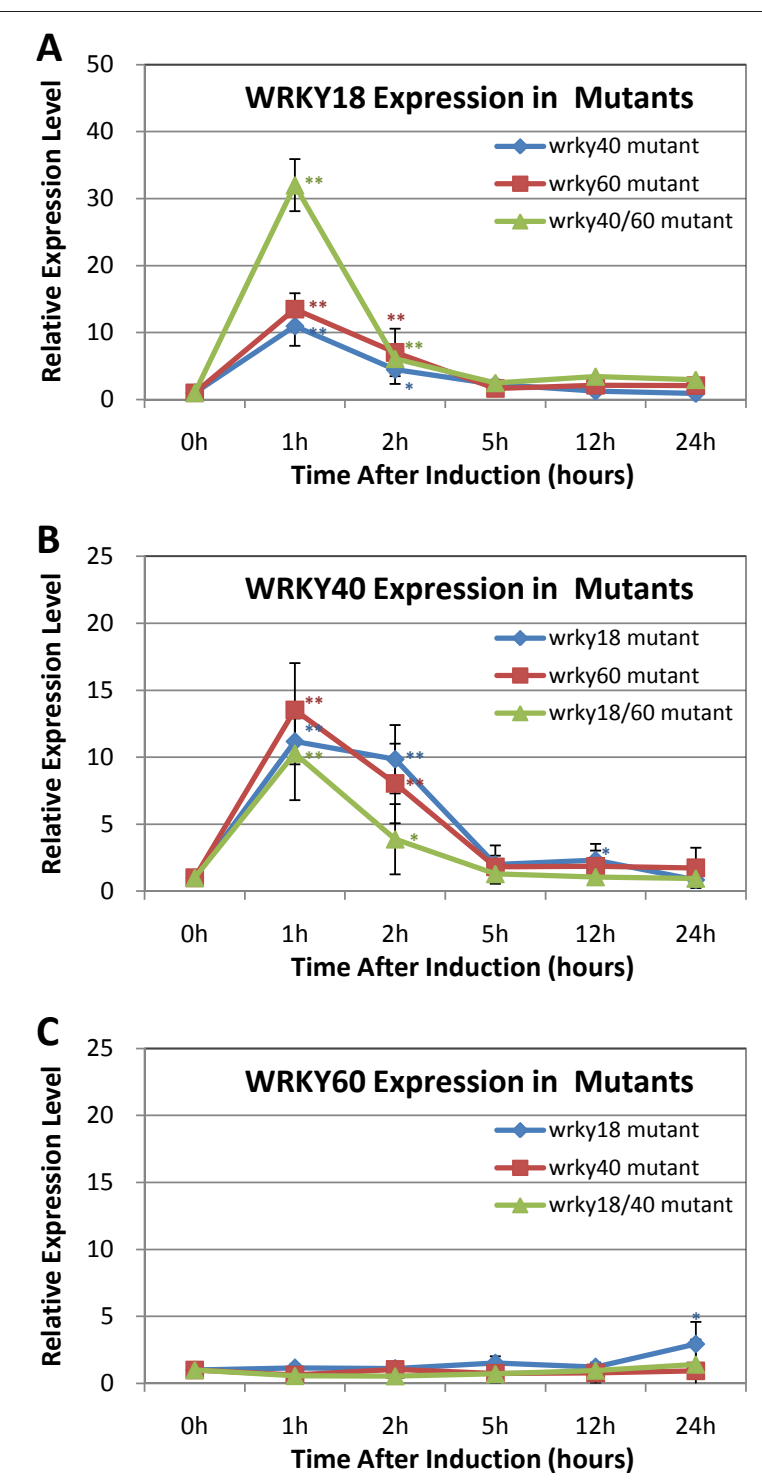

Figure 5 WRKY18- and WRKY40-dependency of ABA-induced expression of WRKY60. Three-weeks-old wild-type and mutant plants were sprayed with water (Mock) or $5 \mu \mathrm{M}$ ABA. Leaves from four treated plants were harvested at indicated times after the treatment for isolation of total RNA and analysis of WRKY18 (A), WRKY40 (B) and WRKY60 (C) transcripts using qRT-PCR. Expression level was defined as the ratio of qRT-PCR result of treated sample to its respective mock.

The means and standard errors were calculated from three independent experiments. Asterisks mark statistically significant differences of expression level between ABA-treated-leaves harvested immediately and after indicated time. (Asterisks: p-value $<0.05$; Double Asterisks: p-value < 0.01; by Student-Newman-Keuls Test).

WRKY60 (Figure 4A). Furthermore, ABA induction of WRKY60 was almost completely abolished in the wrky18 and wrky40 mutants (Figure $5 \mathrm{C}$ ). These results suggest that WRKY60 might be directly regulated by WRKY18 and WRKY40. To examine this possibility, we compared the promoters of the three WRKY genes for presence of the TTGACC/T W boxes recognized by WRKY transcription factors. In the $1 \mathrm{~kb}$ promoter regions upstream of the coding sequences, there was a single WRKY box located at 240 bp upstream of the start codon of WRKY18. No TTGACC/T W box was found within the $1.0 \mathrm{~kb}$ upstream promoter sequence of WRKY40. Interestingly, there are three TTGACC/T W box sequences within a 19-bp region from position -791 to position -773 upstream of the translation start site of WRKY60 (Figure 6A). Presence of a cluster of W-boxes in the WRKY60 gene promoter suggests a possible role of

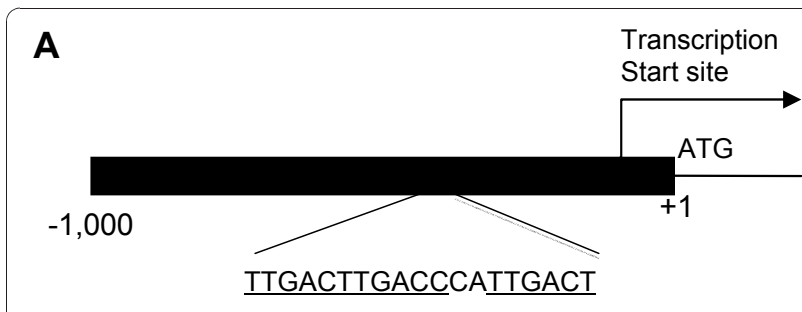

B

PW60: GCTTGACTTGACCCATTGACTATG m P w 0: GCTTGAaTTGAaCCATTGAaTATG

C

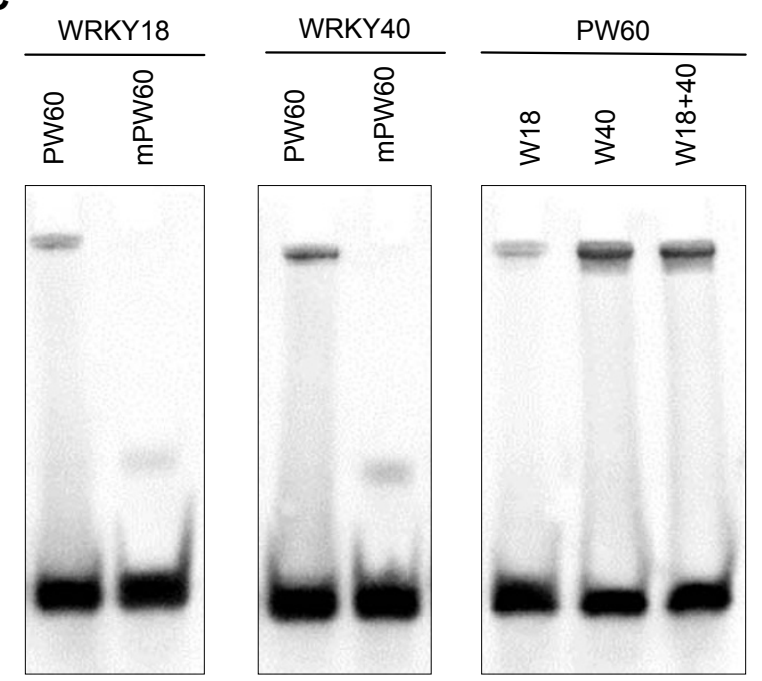

Figure 6 Recognition of the WRKY 60 promoter by WRKY18 and WRKY40. A. Diagram of the WRKY60 gene, including the $1 \mathrm{~kb}$ upstream promoter that contains a cluster of three W-box sequences between -791 and -773 relative to the translation start codon. B. Nucleotide sequences of probes used for EMSA. PW60 contains three TTGAC sequences, which are mutated into TTGAA in mPW60. C. EMSA of binding of PW60 and mPW60 by recombinant WRKY18 protein (labelled as W18), WRKY40 protein (labelled as W40), and their mixture (labelled as W18+40). For each binding assay, $200 \mathrm{fmol}$ recombinant proteins and $20 \mathrm{fmol}$ labeled DNA probe were used. 
WRKY proteins in the regulation of WRKY60 gene expression.

To determine whether the W boxes from the WRKY60 gene promoter are recognized by WRKY18 and WRKY40 proteins, we generated and labelled a double-stranded DNA probe containing these three W boxes (PW60) (Figure 6B). When incubated with recombinant WRKY18 or WRKY40 proteins, the probe produced a retarded band in electrophoretic mobility shift assays (Figure 6C). A similar retarded band was also produced when the probe was incubated with a mixture of WRKY18 and WRKY40 recombinant proteins (Figure 6C). To determine whether the $\mathrm{W}$-boxes in the PW60 probe were important for the recognition, we also tested a mutant probe (mPW60) in which the TTGAC sequence of each W-box was changed to TTGAA (Figure 6B). As shown in Figure $6 \mathrm{C}$, this mutant probe failed to detect retarded bands when incubated with WRKY18 or WRKY40 proteins. Thus, WRKY18 and WRKY40 proteins recognize the W-box sequences in the WRKY60 gene promoter.

\section{Activation of the WRKY60 Promoter by WRKY18 and WRKY40 in Protoplasts}

To determine whether the cluster of $\mathrm{W}$ box sequences are important for ABA-induced expression of WRKY60, we isolated a $\sim 1,000 \mathrm{bp}$ promoter fragment upstream of the translational start of WRKY60 and fused it to the GUS reporter gene (W60:GUS). A mutant WRKY60 promoter, in which the cluster of the W box sequences from position -791 to position -773 upstream of the translation start site of WRKY60 were deleted by overlapping PCR, was also fused to the GUS reporter gene ( $m W 60: G U S$ ). As shown in Figure 7A, addition of ABA into the protoplasts transfected with the W60:GUS construct resulted in about 3.5-fold induction of the reporter gene expression compared with the non-induced condition. On the other hand, addition of ABA into the protoplasts transfected with the mutant $m$ W60:GUS construct resulted in less than 1.5-fold induction of the reporter gene expression compared with the noninduced condition. This result indicated that the $\mathrm{W}$ box sequences are critical for ABA-induced expression of WRKY60.

To determine whether WRKY18 and WRKY40 can activate the WRKY60 promoter in protoplasts, we generated the WRKY18 and WRKY4O effector constructs under control of the constitutive CaMV $35 S$ promoter. As shown in Figure 7B, coexpression of WRKY18 or WRKY40 led to only a very small increase in the reporter gene expression from the W60:GUS construct in the wrky18/wrky40 mutant protoplasts (Figure 7B). On the other hand, coexpression of both WRKY18 and WRKY40 activated the the reporter gene expression the W60:GUS construct by almost 5 -fold in the
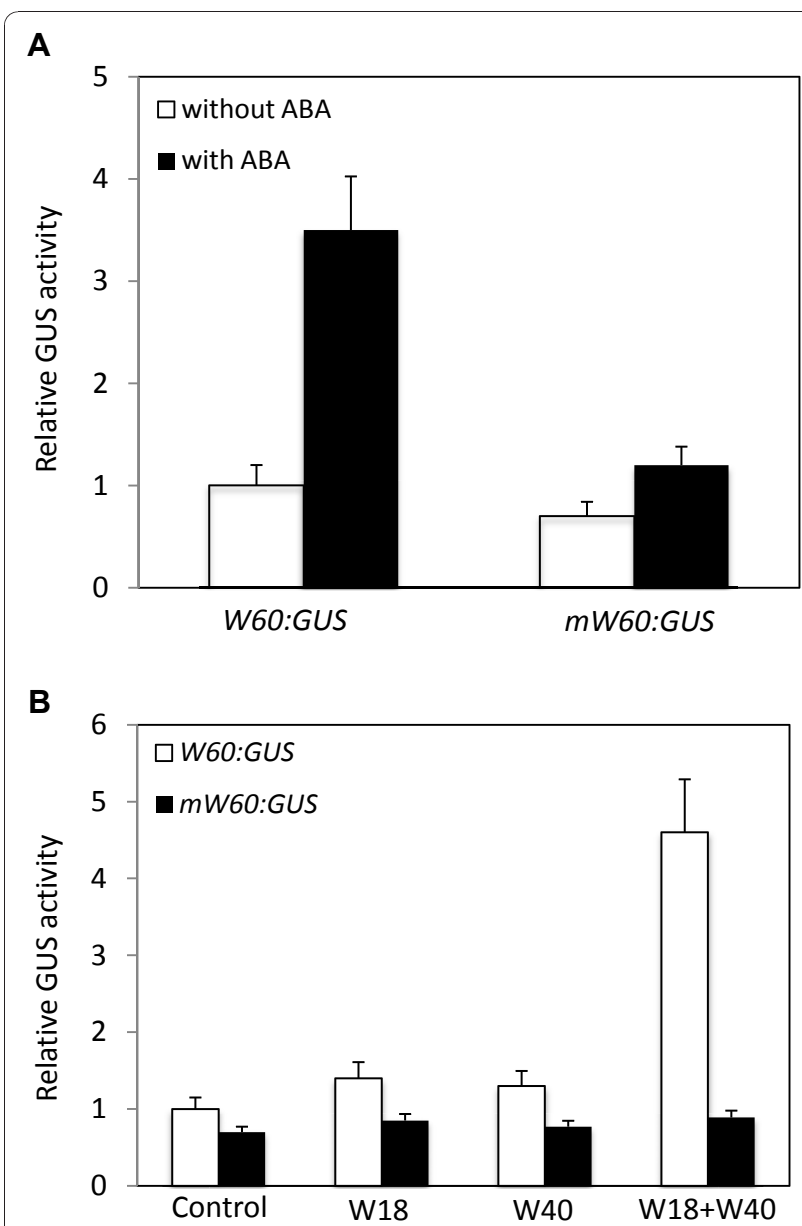

Figure 7 Analysis of the WRKY60:GUS reporter gene using protoplast transfection. A. Effects of ABA and $W$ boxes on the WRKY60 promoter activity. Protoplasts from Col-0 wild type plants were transfected with the GUS reporter gene driven by the WRKY6O promoter (W60:GUS) or a mutant WRKY60 promoter in which the cluster of W-box sequences between -791 and -773 relative to the translation start codon were deleted (mW60:GUS). GUS activities were measured without or $12 \mathrm{~h}$ after the addition of $2 \mu \mathrm{M}$ ABA. B. Effects of co-transfected WRKY18 and WRKY40 on the WRKY60 promoter activity. Protoplasts from wrky $18 /$ wrky 40 double mutant plants were cotransfected with the W60:GUS or mW60:GUS reporter gene and an effect plasmid expressing WRKY18 (W18), or WRKY40 (W40) or two effector plasmids expressing the two WRKY proteins (W18+W40) driven by the WRKY60 promoter (W60:GUS). An empty effector plasmid was used as control. GUS activities were measured $12 \mathrm{~h}$ after co-transfection.

wrky18/wrky40 mutant protoplasts (Figure 7B). This activation of the WRKY60 promoter by coexpression of WRKY18 and WRKY40 was not observed from the $m$ W60:GUS construct (Figure 7B). Thus, WRKY18 and WRKY40 cooperate in the activation of the WRKY6O gene expression mostly likely through recognition of the $\mathrm{W}$ box sequence in the WRKY60 gene promoter. 


\section{Transcription-regulating activity of WRKY18, WRKY40 and WRKY60}

Functional analysis has revealed that structurally related and physically interacting WRKY18, WRKY40 and WRKY60 have a complex pattern of overlapping, antagonistic and distinct roles in plant defense and stress responses [27]. This complex pattern may, in part, result from the distinct transcriptional regulatory activities of the three transcription factors. To test this possibility, we employed a previously established transgenic system to determine the transcriptional regulatory activities of the three WRKY proteins through assays of a reporter gene in stably transformed plants [15]. The reporter gene in the system is a GUS gene driven by a synthetic promoter consisting of the -100 minimal CaMV $35 \mathrm{~S}$ promoter and eight copies of the LexA operator sequence (Figure 8A). Because the minimal $35 S$ promoter is used, transgenic Arabidopsis plants harboring the reporter gene constitutively expressed only low levels of GUS and, therefore, it is possible to assay both transcription activation and repression by determining corresponding increase and decrease in GUS activities following co-expression of an effector protein.

To generate the WRKY18, WRKY40 and WRKY60 effectors, we fused their coding sequences with that of the DNA-binding domain (DBD) of LexA (Figure 8A). The fusion constructs were subcloned behind the steroid-inducible Gal4 promoter in pTA7002 [31] and transformed into transgenic plants that already contain the GUS reporter construct. Unfused WRKY and LexA $D B D$ genes were also subcloned into pTA7002 and transformed into transgenic GUS reporter plants as controls (Figure 8A). For comparison, we also include WRKY48, a strong transcription activator [32], and WRKY7, a transcription repressor [15], in the assays. Transgenic plants containing both the reporter and an effector construct were identified through antibiotic resistance screens. To determine the effect of the effectors on GUS reporter gene expression, we determined the changes of GUS activities in the transgenic plants after induction of the effector gene expression by spraying $20 \mu \mathrm{M}$ dexamethasone (DEX), a steroid. In the transgenic plants that expressed unfused WRKY18, WRKY40, WRKY60 or LexA DBD effector, there were little changes in the GUS activities after 18-hour DEX treatment (Additional file 3). In the transgenic plants harboring the LexA DBD-WRKY18 effector gene, induction of the fusion effector after DEX treatment resulted in 1.4 - fold increase in GUS activity (Additional file 3). A slightly higher 1.6-fold increase in GUS activity was observed in the transgenic plants harboring the LexA $D B D$-WRKY60 effector gene after DEX treatment (Additional file 3). By comparison, as previously reported [32], transgenic plants harboring the LexA DBD-WRKY 48
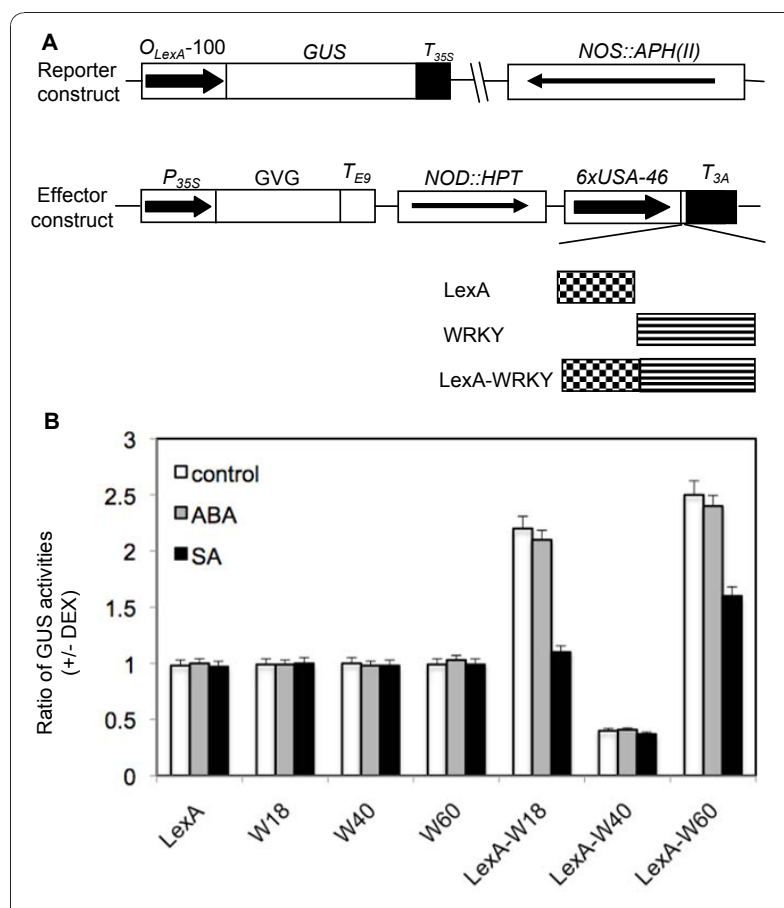

Figure 8 The effect of $A B A$ and $S A$ on the transcriptionregulating activities of WRKY18, WRKY40 and WRKY60.

A. Constructs of reporter and effector genes. The GUS reporter gene is driven by a synthetic promoter consisting of the -100 minimal CaMV 355 promoter and eight copies of the LexA operator sequence. The effector genes were cloned into PTA7002 behind the steroid-inducible promoter. The effector genes encode LexA DBD (LexA), WRKY and LexADBD-WRKY fusion protein, respectively. B. The effect of $A B A$ and $S A$ on the transcription-regulating activity of the WRKY proteins. Progeny from 5 independent transgenic lines for each effector gene were divided into three groups (15-20 plants/ group) and sprayed with DEX $(20 \mu \mathrm{M})$, DEX plus ABA $(10 \mu \mathrm{M})$ or DEX plus SA (1 mM). Leaves were harvested at 0 and 24 hours after the treatment for assays of GUS activities and the ratios of GUS activities were calculated. Only those progeny that displayed induced expression of the effector genes as determined from RNA blotting following DEX treatment were used in the analyses. The means and errors were calculated from at least 15 positive progeny. The experiments were performed twice with similar results.

effector gene, DEX treatment resulted in $\sim 24$-fold increase in GUS activity. These results indicate that both WRKY18 and WRKY60 are weak transcriptional activators. By contrast, in the transgenic plants harboring the LexA DBD-WRKY4O effector gene, induction of the fusion effector after DEX treatment resulted in a 2fold reduction in GUS activity (Additional file 3). In transgenic plants harboring the LexA DBD-WRKY7 effector gene, DEX treatment resulted in $\sim 5$-fold reduction in GUS activity. Thus, WRKY40 is a relatively weak transcriptional repressor.

We have previously shown that WRKY18, WRKY40 and WRKY60 physically interact with themselves and with each other to form both homo- and hetero-complexes 
[27]. In addition, the three WRKY genes are induced by pathogen infection, SA and ABA treatment [27] (Figure 5). Thus, the transcription-regulating activity of the three WRKY proteins may change upon interaction with each other or with other induced proteins. To test this possibility, we examined the effects of SA and ABA treatment on the changes of GUS activities in the progeny of the transgenic effector/reporter lines after 24-hour DEX induction of the effector genes. Extension of DEX treatment from 18 to 24 hours increased significantly the expression levels the effector genes (unpublished data). In the transgenic plants that expressed unfused WRKY18, WRKY40, WRKY60 or LexA DBD effector, there were little changes in the GUS activities after DEX treatment with or without $\mathrm{ABA}$ or SA treatment (Figure $8 \mathrm{~B}$ ). In the transgenic plants harboring the LexA DBD-WRKY18 effector gene, induction of the fusion effector after DEX treatment resulted in 2.2 -fold increase in GUS activity (Figure 8B). ABA treatment had little effect on DEX-induced change of GUS activity, suggesting that ABA did not significantly affect the transcription-activating activity of WRKY18. On the other hand, in SA-treated transgenic plants harboring the LexA DBD-WRKY18 effector gene, there was almost no increase in GUS activity following induction of the fusion effector after DEX treatment. Thus, SA treatment almost completely abolished the transcription-activating activity of WRKY18. In the absence of ABA or SA treatment, a 2.5 -fold increase in GUS activity was observed in the transgenic plants harboring the LexA DBD-WRKY60 effector gene after 24-hour DEX treatment (Figure 8B). Again ABA treatment had little effect on DEX-induced change of GUS activity while SA treatment resulted in more than $50 \%$ reduction in the increase of GUS activity following 24-hour DEX induction of the fused LexA DBD-WRKY60 effector gene (Figure $8 \mathrm{~B}$ ). In the transgenic plants harboring the LexA DBD-WRKY4O effector gene, induction of the fusion effector after DEX treatment resulted in a 2.5fold reduction in GUS activity (Figure 8B). Neither ABA nor SA treatment had significant effect on the change of GUS activities in the transgenic plants harboring the LexA $D B D$-WRKY40 effector gene (Figure 8B). Thus, the transcription-regulating activity of both WRKY18 and WRKY60, but not WRKY40, was substantially altered by SA treatment.

\section{Expression of $A B A$ related genes}

To further understand how the three WRKY proteins are involved in the regulation of ABA responses, we compared wild type and the mutants for the three WRKY mutants for expression of four genes associated with ABA signalling; $A B I 5, A B I 3, S T Z$ and $D R E B 2 A$. As shown in Figure 9 for $A B I 5, S T Z$ and $D R E B 2 A$, we observed no significant difference between the wild type and the mutants when the seedlings were grown in
ABA-less MS grown medium. For $A B I 3$, the basal level were slightly but significantly higher in the $w r k y 18$ and wrky40 mutant plants(Figure 9). On the ABA-containing medium, we observed modest but significant reduction in expression of $S T Z$ in the wrky60 mutant (Figure 9). There was also relatively small reduction in and $S T Z$ expression in the wrky 40 mutant. Surprisingly, no significant reduction of the ABA-related genes was observed in the wrky18 mutant; in fact, there appear to be a small but significant increase in ABA-induced expression of DREB2A in the wrky18 mutant when compared to wild type (Figure 9).

\section{Discussion}

\section{Differential roles of WRKY18, WRKY40 and WRKY60 in $A B A$ and abiotic stress responses}

Over the last several years, there has been growing evidence that plant WRKY transcription factors are involved in plant ABA signaling and abiotic stress responses. In rice and barley, $\mathrm{ABA}$ induces expression of a number of WRKY genes in aleurone cells $[23,24,33,34]$. When transiently overexpressed in aleurone cells, some of these ABA-inducible WRKY genes activate or repress ABA-inducible reporter genes. A number of studies have also shown that WRKY genes are induced by a variety of abiotic stress conditions and overexpression of some WRKY genes altered plant stress tolerance. In the present study, we have determined the role of three Arabidopsis WRKY genes in plant ABA signaling by analyzing the effects of ABA on germination, root growth of their knockout mutants and overexpression lines. We have demonstrated that while disruption of WRKY18 and WRKY60 caused reduced sensitivity to ABA, disruption of WRKY40 increased ABA sensitivity for inhibition of germination and root growth (Figures 1 and 2). Likewise, we have demonstrated that the wrky18 and wrky60 mutants but not the wrky40 mutant are more tolerant to salt and osmotic stress (Figure 3). The differential roles of the three structurally related WRKY proteins in plant ABA and abiotic stress responses were also demonstrated from the analysis of the double and triple knockout mutants and overexpression lines (Figure 1, 2 and 3).

The role of ABA during seed germination has been extensively studied. The opposite phenotypes of the wrky mutants in ABA sensitivity for inhibition of germination strongly suggest that these WRKY genes function as either positive or negative regulators of ABA signaling. Although no altered phenotypes of the wrky 40 mutant was observed in ABA effects on root growth or salt and osmotic sensitivity, which could be due to low sensitivity of the assays, we did observe that the wrky18 and wrky60 mutants exhibited reduced ABA inhibition of root growth as well as reduced sensitivity to salt and 

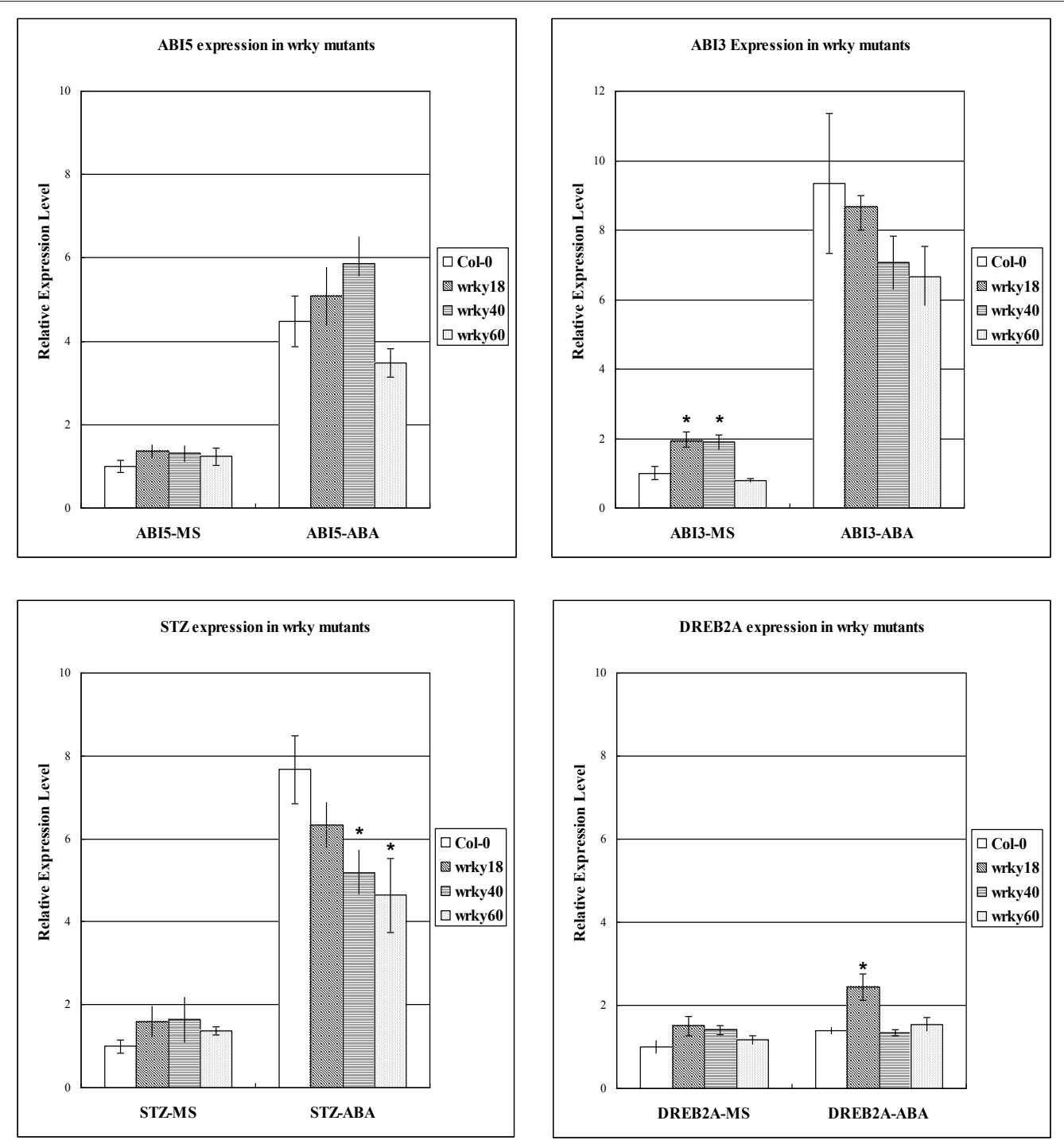

Figure 9 RNA levels of $A B I 3, A B I 5, D R E B 2 A$ and $S T Z$ in wrky18, 40, 60 mutants and wild type seedlings. Seedlings of wild type or mutants were grown on MS medium for 14 days before being transplanted onto MS plates with or without $2.0 \mu \mathrm{M}$ ABA. RNA was extracted from seedlings on MS medium 12 hours after transplantation. Relative RNA levels of the 4 genes ABI3, ABI5, DREB2A and STZ were analyzed using gene-specific primers by real-time PCR. The means and standard errors were calculated from three independent experiments, all of which included no less than 20 seedlings per sample. Asterisks mark statistically significant differences of expression level between genotypically identical seedlings with or without ABA treatment, by Student-Newman-Keuls Test(p-value $<0.05)$.

osmotic stress (Figure 1, 2 and 3). Therefore, it is possible that altered phenotypes in abiotic stress are related to altered ABA signaling in the WRKY gene mutants. For example, the higher level of DREB2A in wrky 18 mutant than in wild type plants under exogenous ABA treatment may partially explain the higher abiotic resis$\operatorname{tanc}($ Figure 12, 3 and 9), considering overexpression of transcriptional activation domain of DREB2A resulted in significant drought stress tolerance [35]. It is known that the inhibited effect of ABA on root growth involves pathways mediated by other plant hormones such as ethylene, auxin and jasmonic acid. The relationship between ABA signaling and salt and osmotic stress tolerance is also very complex. In some mutants such as tomato tss 2 mutant, ABA hypersensitivity is associated with osmotic stress hypersensitivity [36,37]. In other mutants such as the tos mutant, ABA insensitivity is associated with osmotic stress hypersensitivity [38]. These studies suggest that proper levels of ABA perception and signaling are important for the abiotic stress tolerance. WRKY18 and WRKY60 are weak transcriptional activators and WRKY40 is a weak transcriptional repressor (Figure 8). The relatively weak transcription regulatory activities would make the three transcription 
factors suitable as either positive or negative regulators for modulating ABA signaling and influencing ABA-regulated plant growth and abiotic stress responses (Figure 10).

The roles of WRKY18, WRKY40 and WRKY60 in ABA signaling are consistent with the ABA-inducible expression of the three genes (Figure 4). Interestingly, the three WRKY genes display distinct expression patterns upon ABA treatment. WRKY18 and WRKY4O are rapidly induced upon ABA treatment and are required for ABAinduced WRKY60 expression (Figure 4). On the other hand, ABA-induced expression of WRKY 60 is delayed but also prolonged (Figure 4). In addition, WRKY60 and WRKY4O act partially redundantly in repressing WRKY18 expression (Figure 5). This expression pattern raises the possibility that the three WRKY proteins are part of a regulatory network that modulates gene expression in the ABA signaling pathway. Upon ABA induction, WRKY18 and WRKY4O are first induced and their

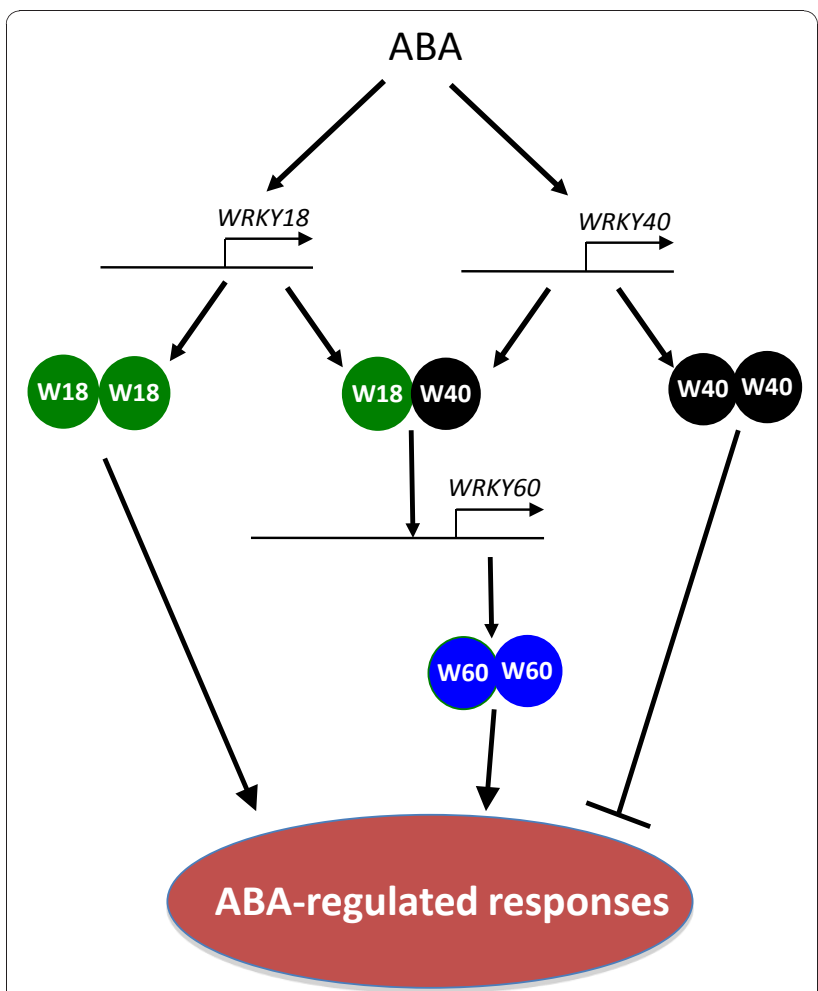

Figure 10 Proposed model for involvement of WRKY18, WRKY40 and WRKY60 in ABA responses. ABA induction of WRKY18 and WRKY40 leads to increase in WRKY18 and WRKY40 proteins that form both homo- and heterocomplexes through physical interactions. The requirement of both WRKY18 and WRKY40 for induction of WRKY60 suggest possible involvement of a WRKY18/WRKY40 heterocomplex that may recognize the $W$ box sequences in the WRKY60 gene promoter and activate its expression. WRKY18 and WRKY60 positively regulate while WRKY40 negatively regulates plant responses to $A B A$ probably by modulating ABA-regulated genes. products could act as early transcriptional effectors to regulate expression of additional ABA-induced genes including WRKY60 (Figure 10). Induced WRKY60 would then act with WRKY40 to repress WRKY18, forming a negative feedback loop. The prolonged expression and the transcription-activating activity of WRKY60 would allow it to have a relatively sustained effect on ABAregulated gene expression. This interpretation is consistent with the relatively strong phenotypes of the wrky60 mutant in ABA and stress tolerance when compared to those of the wrky18 mutant (Figure 1,2 and 3).

\section{Roles of WRKY18, WRKY40 and WRKY60 in crosstalk between abiotic and biotic responses}

We have previously shown that single wrky18, wrky40 and wrky60 mutants exhibited no or small alterations in response to the hemibiotrophic bacterial pathogen $P$. syringae or the necrotrophic fungal pathogen B. cinerea [27]. However, wrky18 wrky 40 and wrky18 wrky60 double mutants and the wrky 18 wrky 40 wrky60 triple mutant were substantially more resistant to $P$. syringae but more susceptible to $B$. cinerea than wild-type plants [27]. These phenotypes and additional analysis of SA- and JA-regulated gene expression suggest that these WRKY proteins have a partially redundant negative effect on SA-mediated defense but exerted a positive role in JA-mediated defense. Likewise, we have shown in this report that WRKY18 and WRKY60 positively regulate while WRKY40 negatively regulates plant ABA response (Figure 1, 2 and 3). As ABA is known to counteract SA-defense [7] but function as a signal in JAmediated defense against necrotrophic pathogens [28], the roles of these three WRKY proteins in plant defense and $A B A$ and stress responses might be mechanistically linked. This notion is particularly attractive for WRKY18 and WRKY60, which might negatively impact SA-dependent defense through positively modulating ABA signaling. On the other hand, WRKY40 antagonized WRKY18 and WRKY60 in ABA response but functions partially redundantly with WRKY18 and WRKY60 in SA-dependent defense. As will be discussed later, WRKY18, WRKY40 and WRKY60 interact with themselves and with each other to form distinct complexes that may differ in both DNA-binding and transcription-regulating activities. The interacting partners of WRKY40 formed during pathogen infection might not be the same as those in ABA-treated plants and, therefore, may function in distinct manners during plant defense and stress responses.

\section{Molecular basis of functional interactions among WRKY18, WRKY40 and WRKY60}

We have previously shown that through a leucine-zipper motif present at the $\mathrm{N}$-terminus of the three proteins, 
WRKY18, WRKY40 and WRKY60 interacts with themselves and with each other to form both homo-complexes and hetero-complexes with altered DNA binding activities [27]. In the present study, we have shown that WRKY18 and WRKY60 act as weak transcriptional activators and WRKY40 is a transcriptional repressor in plant cells (Figure 8). Furthermore, we have shown that SA treatment can diminish or reduce the transcriptionactivating activity of WRKY18 and WRKY60 (Figure 8). Thus, the three WRKY proteins may form a range of protein complexes with distinct DNA-binding and transcription-activating or -repressing activities. The complex pattern of DNA binding and transcription regulatory activities of the three WRKY proteins may explain their complex biological roles in plant defense and stress responses.

In plant defense responses, analysis of T-DNA insertion mutants indicated that WRKY18, WRKY40 and WRKY60 have redundant repressor function in plant defense against virulent hemibiotrophic P. syringae and biotrophic Golovinomyces orontii [27,39]. Genome-wide gene expression profiling experiments also showed that WRKY18 and WRKY40 have a redundant role in repressing a subset of 23 genes associated with PAMP-triggered immunity [39]. The redundant roles of WRKY18 and WRKY40 as repressors of plant defense genes are consistent with the demonstrated repressing activity of WRKY40 but not with the transcription-activating activity of WRKY18. However, we have also shown that after treatment with SA, which is elevated in pathogen-infected plants, the transcriptionactivating activity of WRKY18 is largely diminished (Figure 8). Under such conditions WRKY18 may compete for binding to promoter sequences with other pathogeninduced WRKY proteins with stronger transcription-activating activities, thereby preventing strong expression of the target genes. In the absence of SA treatment or pathogen infection, on the other hand, WRKY18 may function as a positive regulator of plant disease resistance by acting as an activator of plant defense genes as observed in transgenic WRKY18-overexpressing plants [40]. The positive role of WRKY18 as a positive regulator of disease resistance and activator of defense gene would be antagonized by the transcription-repressing WRKY40 if they are coexppressed. Indeed, we have previously observed that potentiated defense responses in WRKY18-overexpressing Arabidopsis plants are abolished by co-overexpression of WRKY40 in the same transgenic plants [40].

The differential roles of the three WRKY proteins in plant responses to $\mathrm{ABA}$ and abiotic stress conditions are correlated with their distinct transcriptional regulatory activities. WRKY18 and WRKY60 act as transcriptional activators and functional as positive regulators of plant $\mathrm{ABA}$ and abiotic stress responses. By contrast, WRKY40 acts as a transcriptional repressor and functional as a negative regulator of plant $\mathrm{ABA}$ responses. Thus, it is mostly likely that the roles of the three WRKY proteins in plant ABA and stress responses are mediated by their activities in activating or repressing plant genes involved in $\mathrm{ABA}$ and stress signaling.

ABA-induced expression of WRKY60 is severely compromised in both the wrky18 and wrky40 single mutants (Figure 6C). Thus, both WRKY18 and WRKY40 are important for ABA-induced WRKY60 expression. In the promoter of WRKY60, there is a cluster of three W boxes within a $19 \mathrm{bp}$ region (Figure 6A), which are important for ABA-induced expression of WRKY60 in protoplasts (Figure 7A). Using EMSA, we have shown that the cluster of W boxes in the WRKY60 gene promoter is recognized by both WRKY18 and WRKY40 (Figure 6C). Protoplast transfection assays further showed that only co-overexpression of WRKY18 and WRKY40 but not WRKY18 or WRKY40 alone led to activation of the WRKY60 gene promoter and this activation of WRKY60 was dependent on the cluster of three $\mathrm{W}$ boxes in its promoter (Figure 7). It is possible that upon ABA treatment, WRKY18 and WRKY40 are first induced and cooperative binding of induced WRKY18 and WRKY40 or binding of a WRKY18/ WRKY40 heterocomplex to the cluster of W boxes in the WRKY60 promoter is necessary for the subsequent induction of WRKY60 (Figure 10).

\section{Conclusions}

We have found that mutants and overexpression lines for Arabidopsis WRKY18, WRKY40 and WRKY60 genes have altered phenotypes in plant sensitivity to ABA, salt and osmotic stress. Thus, the three WRKY transcription factors play roles in both plant biotic and abiotic stress responses. Additional studies of their expression, DNA binding and transcription-regulating activities strongly suggest that the three WRKY transcription factors form a highly interacting regulatory network that modulates gene expression in both plant defense and stress responses.

\section{Methods}

\section{Materials and Growth Conditions}

The Arabidopsis knockout mutants and overexpression lines for WRKY18, WRKY4O and WRKY60 have been previously described [27]. The plants were grown in mixture of peat/forest soil (purchased from Pingstrup Substrate) and vermiculite (3:1) in a green house at $23^{\circ} \mathrm{C}$ with $150 \mu \mathrm{E} \mathrm{m}^{-2} \mathrm{~s}^{-1}$ light on a photoperiod of $12 \mathrm{~h}$ light and $12 \mathrm{~h}$ dark.

\section{Assays of Sensitivity to ABA and Stress}

Seeds (100 seeds for each replicate) of wild type, mutants and overexpression lines were surface sterilized by treating for $5 \mathrm{~min}$ in $15 \%$ bleach and $0.5 \%$ Tween-20. The 
sterilized seeds were placed on 1/2 Murashige and Skoog medium (Gibcol) and $0.3 \%$ phytagel (Sigma) and stratified at $4^{\circ} \mathrm{C}$ for 4 days before transfer to $23^{\circ} \mathrm{C}$ for germination and growth. For tests of the ABA effect on germination, seeds were plated directly onto media containing various concentrations of $\mathrm{ABA}$. For testing root elongation under $\mathrm{ABA}$ or abiotic stress treatments, seeds were firstly germinated on MS media. Four-days-old seedlings were then transferred to media containing ABA, mannitol, PEG or $\mathrm{NaCl}$. Root length was measured 7 days after transfer using the NIH ImageJ1.41 program.

\section{Cloning, expression, purification of recombinant proteins and the EMSA}

Cloning, expression in E. coli and purification of recombinant WRKY 18 and 40 proteins have been previously described [27]. 5' biotin labeled DNA probes of the WRKY60 promoter was synthesized by Invitrogen. EMSA and detection were performed according to the manual of the Pierce's Lightshift Chemiluminescent EMSA Kit. In each binding assay, 200 fmol recombinant WRKY protein and 20 fmol DNA probe were used.

\section{Gene expression analysis}

Total RNA was extracted from plant samples following the instructions in handbook of Trizol (Invitrogen) and treated with RNase-free DNase I (Promega) to remove contaminated DNA. cDNA was synthesized by adding $100 \mathrm{ng}$ total RNA into $10 \mu \mathrm{l}$ reaction with random hexames and oligo dT primers provided by PrimeScript RT Reagent Kit (Takara). Quantitative-real time PCR was performed in ABI7900 HT machine with SYBR PrimeScript RT-PCR Kit (Takara). The RT-reaction product $(2 \mu \mathrm{l})$ was used as template in a $25 \mu \mathrm{l} \mathrm{PCR}$ mixture. The following program was used for PCR amplification: Initial denaturation at $95^{\circ} \mathrm{C} 10 \mathrm{sec}$. followed by 40 cycles of $95^{\circ} \mathrm{C} 5 \mathrm{sec}$. and $60^{\circ} \mathrm{C} 30 \mathrm{sec}$. The $\beta$-actin gene was used as endogenous reference gene. Data analysis was performed using the ABI SDS 2.0 program. The primers used in real time PCR are listed in Additional file 4.

\section{Protoplast transfection assays}

The full-length GUS gene was clone into the XbaI site of pFF19 [41]. The $1.0 \mathrm{~kb}$ WRKY60 promoter was PCR-amplified using the following two primers: atgcaagcTTTCTTTGTTTTCTGCCGGTTT and atgcgagctcAAATTTAGGTTCACAGGAGCCA. The amplified promoter DNA was digested with HindIII and SacI and was used to replace the CaMV $35 S$ promoter in pFF19. The mutant WRKY60 promoter in which the cluster of W-box sequences between -791 and -773 relative to the translation start codon was generated by overlapping PCR. The sequences of the promoters were verified by DNA sequencing.
To generate the WRKY18 and WRKY4O effector constructs, their cDNA fragments that contained the full coding sequences and the 3'-untranslated regions were excised from their respective cloning plasmids and subcloned into the same restriction sites of pFF19 in the sense orientation behind the $35 \mathrm{~S}$ promoter.

Protoplast isolation and transfection were carried out according to the protocols as previously described [42]. Four- to five-weeks old rosette leaves were used for isolation of mesophyll protoplasts. Protoplast transfection was performed using $40 \%$ polyethylene glycol with 10 $\mu \mathrm{g}$ reporter plasmid and $15 \mu \mathrm{g}$ effector plasmid DNA.

\section{Assays of Transcriptional Regulatory Activity}

Transgenic Arabidopsis plants containing a GUS reporter gene driven by a synthetic promoter consisting of the -100 minimal CaMV $35 S$ promoter and eight copies of the LexA operator sequence were previously described [15]. To generate effector genes, the DNA fragment for the LexA DBD was digested from the plasmid pEG202 (Clontech) using HindIII and EcoRI and cloned into the same sites in pBluescript. The full-length WRKY18, WRKY40 and WRKY60 cDNA fragments were subsequently subcloned behind the $\operatorname{Lex} A D B D$ to generate translational fusions. The LexA DBD-WRKY fusion genes were cloned into the XhoI and SpeI site of pTA2002 behind the steroid-inducible promoter [31]. As controls, the unfused LexADBD and WRKY genes were also cloned into the same sites of PTA7002. These effector constructs were directly transformed into the transgenic GUS reporter plants and double transformants were identified through screening for antibiotic (hygromycin) resistance. Determination of activation or repression of GUS reporter gene expression by the effector proteins was performed as previously described [15]. For determining the effect of $A B A$ and SA on the transcription-regulating activity of the WRKY proteins, progeny from 5 independent transgenic lines for each effector gene were divided into three groups (15-20 plants/group) and sprayed with DEX $(20 \mu \mathrm{M})$, DEX plus ABA $(10 \mu \mathrm{M})$ or DEX plus SA $(1 \mathrm{mM})$. Leaves were harvested at 0 and 24 hours after the treatment for assays of GUS activities.

\section{Additional material}

Additional file 1: Altered germination rates under exogenous $A B A$ treatment. Seeds of wild type, mutants and overexpression lines were sown on 1/2 MS media containing indicated concentrations of ABA. Seedlings with green cotyledons were considered as germinated.

Additional file 2: Altered stress tolerance of the WRKY mutants. Seeds of wild type and mutants were grown on 1/2 MS media for four days and then were transferred to MS agar media without or with -0.75 MPa PEG, $200 \mathrm{mM}$ mannitol or $150 \mathrm{mM} \mathrm{NaCl}$. The picture was taken and the root length was determined at the 7th day after the transfer. The average root length of each genotype in MS medium and their standard errors were calculated from three independent experiments, every of 
each employed more than 25 seedlings per genotype. Relative root length was the ratio of average root lengths of seedlings in medium with $-0.75 \mathrm{MPa}$ PEG, $200 \mathrm{mM}$ mannitol or $150 \mathrm{mM} \mathrm{NaCl}$ to those in MS medium.

Additional file 3: Transcription-regulating activities of WRKY18, WRKY40 and WRKY60. The ratios of GUS activities were calculated from the GUS activities determined in the leaves harvested 18 hours after DEX treatment $(+)$ over those determined prior to DEX treatment (-). Only those transformants that displayed induced expression of the effector genes as determined from RNA blotting following DEX treatment were used in the analyses. The means and errors were calculated from at least 15 positive transformants. The experiments were performed twice with similar results.

Additional file 4: Primer sequences for qRT-PCR assay. The designs of these primers were based on mRNA sequence of from At $4 \mathrm{~g} 31800$, At1g80840, At2g25000, AT5G05410.1, AT1G27730, AT2G36270 and AT3G24650 respectively and generated single sharp peeks in melt curves.

\section{Acknowledgements}

We thank Guangdong Natural Science Foundation (grant no. 06023150) and National Science and Technology Major Projects (grant no. 2009ZX08001016B) (CN) for supporting this research to XX and US National Science Foundation grant MCB-0209819 to ZC.

\section{Author details}

${ }^{1}$ State Key Laboratory of Biocontrol and Key Laboratory of Gene Engineering of the Ministry of Education, School of Life Sciences, Sun Yat-sen University, Guangzhou 510275, China. ${ }^{2}$ Department of Botany and Plant Pathology, Purdue University, West Lafayette, IN 47907-2054, USA.

\section{Authors' contributions}

HC performed major part of phenotype assays, expression assay and EMSA assay and composed the draft. LZ analyzed the activator/repressor activity of the WRKY proteins. JS composed the draft with $\mathrm{HC}$ and help in studying the phenotypes. YX helped analyzing expression patterns. ZC participated in the design of the study and edited the manuscript. XX conceived of the study, participated in the design of the study and edited the manuscript. All authors read and approved the final manuscript.

Received: 11 September 2009 Accepted: 19 December 2010 Published: 19 December 2010

\section{References}

1. Cao H, Glazebrook J, Clarke JD, Volko S, Dong X: The Arabidopsis NPR1 gene that controls systemic acquired resistance encodes a novel protein containing ankyrin repeats. Cell 1997, 88(1):57-63.

2. Wildermuth MC, Dewdney J, Wu G, Ausubel FM: Isochorismate synthase is required to synthesize salicylic acid for plant defence. Nature 2001, 414(6863):562-565

3. Glazebrook J: Contrasting Mechanisms of Defense Against Biotrophic and Necrotrophic Pathogens. Annu Rev Phytopathol 2005, 43:205-227.

4. Verslues PE, Zhu JK: New developments in abscisic acid perception and metabolism. Curr Opin Plant Biol 2007, 10(5):447-452.

5. Xiong L, Ishitani M, Zhu JK: Interaction of osmotic stress, temperature, and abscisic acid in the regulation of gene expression in Arabidopsis. Plant Physiol 1999, 119(1):205-212.

6. Asselbergh B, De Vleesschauwer D, Hofte M: Global switches and finetuning-ABA modulates plant pathogen defense. Mol Plant Microbe Interact 2008, 21(6):709-719.

7. Fujita M, Fujita Y, Noutoshi Y, Takahashi F, Narusaka Y, YamaguchiShinozaki K, Shinozaki K: Crosstalk between abiotic and biotic stress responses: a current view from the points of convergence in the stress signaling networks. Curr Opin Plant Biol 2006, 9(4):436-442.

8. Dong J, Chen C, Chen Z: Expression profile of the Arabidopsis WRKY gene superfamily during plant defense response. Plant Mol Biol 2003, 51:21-37.
9. Yu D, Chen C, Chen Z: Evidence for an important role of WRKY DNA binding proteins in the regulation of NPR1 gene expression. Plant Cell 2001, 13(7):1527-1540

10. Li J, Brader G, Palva ET: The WRKY70 transcription factor: a node of convergence for jasmonate-mediated and salicylate-mediated signals in plant defense. Plant Cell 2004, 16(2):319-331.

11. Li J, Brader G, Kariola T, Palva ET: WRKY70 modulates the selection of signaling pathways in plant defense. Plant J 2006, 46(3):477-491.

12. AbuQamar S, Chen X, Dhawan R, Bluhm B, Salmeron J, Lam S, Dietrich RA, Mengiste T: Expression profiling and mutant analysis reveals complex regulatory networks involved in Arabidopsis response to Botrytis infection. Plant J 2006, 48(1):28-44.

13. Zheng Z, Qamar SA, Chen Z, Mengiste T: Arabidopsis WRKY33 transcription factor is required for resistance to necrotrophic fungal pathogens. Plant J 2006, 48(4):592-605.

14. Park CY, Lee JH, Yoo JH, Moon BC, Choi MS, Kang YH, Lee SM, Kim HS, Kang KY, Chung WS, Lim CO, Cho MJ: WRKY group Ild transcription factors interact with calmodulin. FEBS Lett 2005, 579(6):1545-1550.

15. Kim KC, Fan B, Chen Z: Pathogen-Induced Arabidopsis WRKY7 Is a Transcriptional Repressor and Enhances Plant Susceptibility to Pseudomonas syringae. Plant Physiol 2006, 142(3):1180-1192.

16. Journot-Catalino N, Somssich IE, Roby D, Kroj T: The Transcription Factors WRKY11 and WRKY17 Act as Negative Regulators of Basal Resistance in Arabidopsis thaliana. Plant Cell 2006, 18(11):3289-3302.

17. Kim KC, Lai Z, Fan B, Chen Z: Arabidopsis WRKY38 and WRKY62 transcription factors interact with histone deacetylase 19 in basal defense. Plant Cell 2008, 20(9):2357-2371.

18. Seki M, Narusaka M, Ishida J, Nanjo T, Fujita M, Oono Y, Kamiya A, Nakajima M, Enju A, Sakurai T, Akiyama K, Taji T, Yamaguchi-Shinozaki K, Carninci P, Kawai J, Hayashizaki Y, Shinozaki K: Monitoring the expression profiles of 7000 Arabidopsis genes under drought, cold and high-salinity stresses using a full-length cDNA microarray. Plant I 2002, 31(3):279-292.

19. Fowler S, Thomashow MF: Arabidopsis transcriptome profiling indicates that multiple regulatory pathways are activated during cold acclimation in addition to the CBF cold response pathway. Plant Cell 2002, 14(8):1675-1690

20. Pnueli L, Hallak-Herr E, Rozenberg M, Cohen M, Goloubinoff P, Kaplan A, Mittler R: Molecular and biochemical mechanisms associated with dormancy and drought tolerance in the desert legume Retama raetam. Plant J 2002, 31(3):319-330.

21. Mare C, Mazzucotelli E, Crosatti C, Francia E, Stanca AM, Cattivelli L: HvWRKY38: a new transcription factor involved in cold- and droughtresponse in barley. Plant Mol Biol 2004, 55(3):399-416.

22. Rizhsky L, Liang H, Mittler R: The combined effect of drought stress and heat shock on gene expression in tobacco. Plant Physiol 2002, 130(3):1143-1151.

23. Zhang ZL, Xie Z, Zou X, Casaretto J, Ho TH, Shen QJ: A rice WRKY gene encodes a transcriptional repressor of the gibberellin signaling pathway in aleurone cells. Plant Physiol 2004, 134(4):1500-1513.

24. Xie Z, Zhang ZL, Zou X, Huang J, Ruas P, Thompson D, Shen QJ: Annotations and functional analyses of the rice WRKY gene superfamily reveal positive and negative regulators of abscisic acid signaling in aleurone cells. Plant Physiol 2005, 137(1):176-189.

25. Zhou QY, Tian AG, Zou HF, Xie ZM, Lei G, Huang J, Wang CM, Wang HW, Zhang JS, Chen SY: Soybean WRKY-type transcription factor genes, GmWRKY13, GmWRKY21, and GmWRKY54, confer differential tolerance to abiotic stresses in transgenic Arabidopsis plants. Plant Biotechnol J 2008, 6(5):486-503.

26. Jiang W, Yu D: Arabidopsis WRKY2 transcription factor mediates seed germination and postgermination arrest of development by abscisic acid. BMC Plant Biol 2009, 9:96.

27. Xu X, Chen C, Fan B, Chen Z: Physical and functional interactions between pathogen-induced Arabidopsis WRKY18, WRKY40, and WRKY60 transcription factors. Plant Cell 2006, 18(5):1310-1326.

28. Adie BA, Perez-Perez J, Perez-Perez MM, Godoy M, Sanchez-Serrano JJ, Schmelz EA, Solano R: ABA is an essential signal for plant resistance to pathogens affecting JA biosynthesis and the activation of defenses in Arabidopsis. Plant Cell 2007, 19(5):1665-1681.

29. Kilian J, Whitehead D, Horak J, Wanke D, Weinl S, Batistic O, D'Angelo C, Bornberg-Bauer E, Kudla J, Harter K: The AtGenExpress global stress 
expression data set: protocols, evaluation and model data analysis of UV-B light, drought and cold stress responses. Plant J 2007, 50(2):347-363.

30. Seki M, Ishida J, Narusaka M, Fujita M, Nanjo T, Umezawa T, Kamiya A, Nakajima M, Enju A, Sakurai T, Satou M, Akiyama K, Yamaguchi-Shinozaki K, Carninci P, Kawai J, Hayashizaki Y, Shinozaki K: Monitoring the expression pattern of around 7,000 Arabidopsis genes under ABA treatments using a full-length cDNA microarray. Funct Integr Genomics 2002, 2(6):282-291.

31. Aoyama T, Chua N-H: A glucocorticoid-mediated transcriptional induction system in transgenic plants. Plant J 1997, 11:605-612.

32. Xing D, Lai Z, Zheng Z, Vinod KM, Fan B, Chen Z: Stress- and pathogeninduced Arabidopsis WRKY48 is a transcriptional activator that represses plant basal defense. Mol Plant 2008, 1(3):459-470.

33. Xie Z, Zhang ZL, Hanzlik S, Cook E, Shen QJ: Salicylic acid inhibits gibberellin-induced alpha-amylase expression and seed germination via a pathway involving an abscisic-acid-inducible WRKY gene. Plant Mol Biol 2007, 64(3):293-303.

34. Xie Z, Zhang ZL, Zou X, Yang G, Komatsu S, Shen QJ: Interactions of two abscisic-acid induced WRKY genes in repressing gibberellin signaling in aleurone cells. Plant J 2006, 46(2):231-242.

35. Sakuma Y, Maruyama K, Osakabe Y, Qin F, Seki M, Shinozaki K, YamaguchiShinozaki K: Functional Analysis of an Arabidopsis Transcription Factor, DREB2A, Involved in Drought-Responsive Gene Expression. Plant Cell 2006, 18(5):1292-1309.

36. Borsani O, Cuartero J, Fernandez JA, Valpuesta V, Botella MA: Identification of two loci in tomato reveals distinct mechanisms for salt tolerance. Plant Cell 2001, 13(4):873-887.

37. Rosado A, Amaya I, Valpuesta V, Cuartero J, Botella MA, Borsani O: ABAand ethylene-mediated responses in osmotically stressed tomato are regulated by the TSS2 and TOS1 loci. J Exp Bot 2006, 57(12):3327-3335.

38. Borsani O, Cuartero J, Valpuesta V, Botella MA: Tomato tos1 mutation identifies a gene essential for osmotic tolerance and abscisic acid sensitivity. Plant J 2002, 32(6):905-914.

39. Shen QH, Saijo Y, Mauch S, Biskup C, Bieri S, Keller B, Seki H, Ulker B, Somssich IE, Schulze-Lefert P: Nuclear activity of MLA immune receptors links isolate-specific and basal disease-resistance responses. Science 2007, 315(5815):1098-1103.

40. Chen C, Chen Z: Potentiation of developmentally regulated plant defense response by AtWRKY18, a pathogen-induced Arabidopsis transcription factor. Plant Physiol 2002, 129(2):706-716.

41. Timmermans MC, Maliga P, Vieira J, Messing J: The pFF plasmids: cassettes utilising CaMV sequences for expression of foreign genes in plants. $J$ Biotechnol 1990, 14(3-4):333-344.

42. Hwang I, Sheen J: Two-component circuitry in Arabidopsis cytokinin signal transduction. Nature 2001, 413(6854):383-389.

\section{doi:10.1186/1471-2229-10-281}

Cite this article as: Chen et al:: Roles of arabidopsis WRKY18, WRKY40 and WRKY60 transcription factors in plant responses to abscisic acid and abiotic stress. BMC Plant Biology 2010 10:281.

\section{Submit your next manuscript to BioMed Central and take full advantage of:}

- Convenient online submission

- Thorough peer review

- No space constraints or color figure charges

- Immediate publication on acceptance

- Inclusion in PubMed, CAS, Scopus and Google Scholar

- Research which is freely available for redistribution

Submit your manuscript at www.biomedcentral.com/submit 